\title{
Wireless Control of AC Motor using Mesh Network Standard
}

\author{
${ }^{*}$ Aritra De ${ }^{1}$, Ramos-Meza C. ${ }^{2}$, Anup P. Bhat ${ }^{3}$ \\ ${ }^{1}$ Department of Electrical Engineering, University of Engineering \& Management, Jaipur, India \\ ${ }^{2}$ Department of Educational Science, Universidad Andina del Cusco, Cusco, Peru \\ ${ }^{3}$ Department of Electronics, Amolakchand Mahavidyalaya, Yavatmal, Maharashtra, India
}

\begin{abstract}
Nowadays it's very common to use automation in industries. Ac motors are the nerve of many industries. Hence, the automation is much needed for accuracy and reliable operation. This project proposes a system on wireless control and monitoring different parameters of induction motors based on zigbee technology. Due to this, there will be safe data transfer communication in industrial field where the other mode of data transfer communication is more expensive than zigbee or impossible due to some physical conditions. On this design, there will be a set of transducer and sensor monitors the parameters of Induction motor and transmit its data through zigbee. An Arduino based system is used for collecting and storing data and accordingly generating control signal to stop or start the AC machine wireless through computer interface developed with Zigbee. This Techniques has different sensors to monitor and measure different parameters of induction motor and the data is transmitted to control room i.e., drive using zigbee wireless protocol. The overall control like starting and stopping of motor can be done with zigbee that is already interfaced with computer. It also protects motor against some faults such as over current, over heating in winding, under lover voltage, data acquisition system saves all received parameters data of motor in the database.
\end{abstract}

Keywords: Induction motor (IM), Zigbee, Arduino, Current Sensor, Temperature Sensor, voltage sensor, Pc.

\section{I) INTRODUCTION}

Induction motors are mostly preferred in all Industries. The reason for using industrial motor is because, they are selfstarting, economical and reliable. Although they have sufficient features, and simple structure they undergoes several stresses which creates faults which finally ends in failure. To treat these conditions several controlling in motors are needed.

For a reliable operation of motors, they are several data's which plays a major role to control its operation. The data's like current, voltage, temperature and speed decides the performance of Induction motors and drive systems. These parameters directly affects these Motors efficiency and reliability. However, while using Induction motor in Industries, there will be continuous process of motor for these production. On that time if any faults occurs it becomes risky and also very operation to control the motors. The only way for controlling these hazards was to monitor these parameters. It's impossible to monitor these parameters for $24 \times 7$ by humans. So, wireless communication technique for transforming data's from machine to drive is needed, which is used in most of industries.

There are different ways in monitoring Induction motor which was been discovered by many reviewers and researchers. Although, we have different methods in monitoring and controlling Induction motors, still we faces critical problem in Industries, this is because the problem in Induction motors should be found at initial stages. So, that we can rectify it before it creates a large fault. For detection of fault we need fast emerging technology. It should withstand its monitoring and control for all initial faults and also unexpected faults.

Still in most of industries and automation applications, the traditional methods are used i.e., wired technology. Although wired technology operations are designed to meet several hard situations sometimes it creates failures at last. But wired technology was also designed in same way the only difference is that it doesn't have any physical connections. So, that the data communications in wireless manner will be fast as it's compared to wired technology.

In some existing system, there are different methods has been introduced for monitoring, detecting mechanical stresses, controlling temperature in Induction motor. But they didn't fulfill all the controlling and also has some disadvantages, the methodology used will be costlier.

Our proposed system will overcome these defects as we used different protocols like voltage sensors, current sensors, temperature sensors for automatic controlling and interfacing we have used Arduino. In Desktop computer the $\mathrm{C}$ programming were done to design the interface and finally communication. We have used Zigbee methodology which was more efficient than other methods and also cost efficient.

This paper was organized as Section II Wireless Methodology, Section III Proposed. There are different ways in monitoring Induction motor which was been discovered by many reviewers and researchers. Although, we have different methods, this is because the problem in Induction motors should be found at initial stages. So, that we can rectify it before it creates a large fault. For detection of fault we need fast emerging technology. It should withstand its monitoring and control for all initial faults and also unexpected faults. 
Still in most of industries and automation applications, the traditional methods are used i.e., wired technology. Although wired technology operations are designed to meet several hard situations sometimes it creates failures at last. But wired technology was also designed in same way the only difference is that it doesn't have any physical connections. So, that the data communications in wireless manner will be fast as it's compared to wired technology.

In some existing system, there are different methods has been introduced for monitoring, detecting mechanical stresses, controlling temperature in Induction motor. But they didn't fulfill all the controlling and also has some disadvantages, the methodology used will be costlier.

Our proposed system will overcome these defects as we used different protocols like voltage sensors, current sensors, temperature sensors for automatic controlling and interfacing we have used Arduino. In Desktop computer the C programming were done to design the interface and finally communication. We have used Zigbee methodology which was more efficient than other methods and also cost efficient.

This paper was organized as Section II Wireless Methodology, Section III Proposed System, Section IV results, and this paper is finally concluded with Section V.

\section{II) WIRELESS METHODOLOGY}

For remote philosophy the zigbee convention was utilized. Zigbee plays as a transmitter between beneficiary among Drive and Enlistment engine. On IEEE 802.15.4 norm for remote individual territory organizations (WPANs), it is utilized for correspondence of zigbee among control and sensor organization and it is the item from Zigbee partnership. This IEEE standard principally characterizes the correspondence among physical and Media Access Control (Macintosh) layers to deal with the various gadgets with low-information rates.

The WPANs of these sort of zigbee's work at $868 \mathrm{MHz}, 902-928 \mathrm{MHz}$ and $2.4 \mathrm{GHz}$ frequencies. It is most appropriate for intermittent just as middle two path transmission of information among sensors and regulators at the date pace of $250 \mathrm{kbps}$ is. It has three sorts of organization lattice, tree, and star network for these geography associations. The quantity of switches, organizers, and end gadgets relies upon the sort of organization utilized.

\section{Features of Zigbee module:}

* Cost efficient.

* The maximum data rate for a Zigbee device is $250 \mathrm{Kbps}$ because it has low data rate. $\square$ Reliable.

* It can ends upto 65000 nodes in a network.

* It automatically establish it desired network.

* It uses very small packets when has compared to Bluetooth and WIFI.

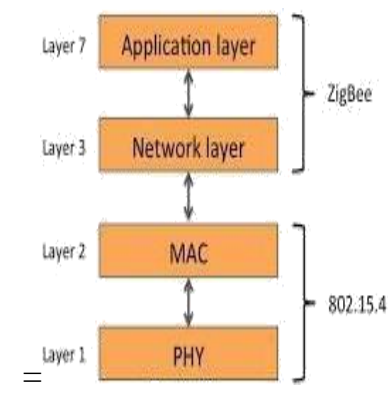

Zigbee specifies itself with three different devices. They are Zigbee Coordinator (ZC), Zigbee Router (RC), Zigbee End devices (ZED).

\section{ZIGBEE COORDINATOR (ZC):}

Zigbee coordinator is one of the devices in Zigbee. It was used for channel selection. It assign an ID for the network. It allocates unique address to each devices and also it initiates and transfer messages to network.

ZIGBEE ROUTER (ZR):

Zigbee Router act as intermediate nodes between the coordinator and the end devices. It router traffic between different nodes. It receives and stores messages intended for their children.

\section{ZIGBEE END DEVICE (ZED):}

Zigbee End devices contains just enough information to talk to the parent node. They may sleep which make end devices more suitable choices for battery operated devices. All traffic to an end devices is first routed to its parent. Examined in two main categories as hardware and software. The functions of the each part of the system are described in the following sections in detail. 


\section{Hardware}

The system consists of a $3 \mathrm{hp} / 1440 \mathrm{rpm}$, T.E.F.C. type three-phase induction motor, and two voltage transformers w

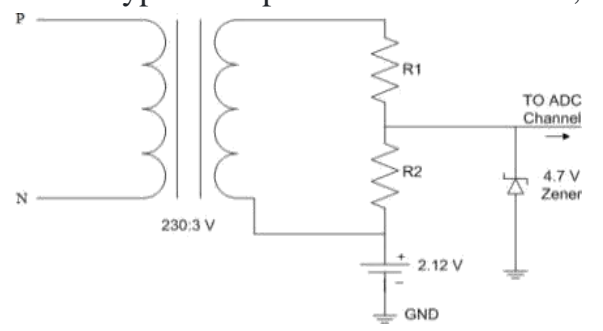

Fig.2: Current Measuring Circuit.

Two signs for voltages, two signs for flows are utilized as contribution to the A/D module of the DSC and one sign for temperature is utilized as advanced contribution to DSC. Fig. 5 and fig. 6 shows the current estimating and voltage estimating circuit individually whose yield is given to the ADC channels of the advanced sign regulator.

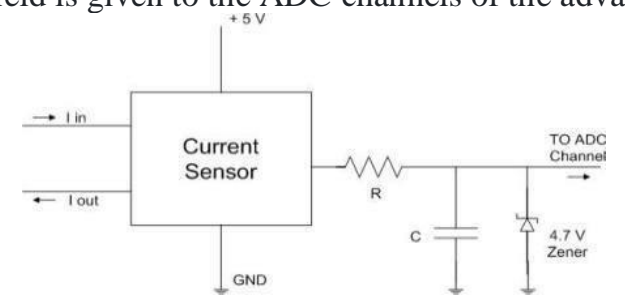

Fig.3: Voltage Measuring Circuit.

The framework programming created in C Programming that works under Windows working framework and has a profoundly adaptable programming structure with information base help. The boundaries of the engine gathered from

\section{III) PROPOSED SYSTEM}

A square graph of proposed is demonstrated as follows. The proposed framework can be sensors dependent on Lobby Impact having Greatest Essential current: $25 \mathrm{~A}$, Auxiliary Voltage (VO): $2.5 \pm 0.625$, recurrence: DC-25 kHz, exactness: $0.1 \%$. Third stage voltage and current is determined by the computerized signal regulator calculation An advanced temperature sensor for engine winding temperature estimating, a 16bit advanced sign regulator dsPIC30F4013 by Central processor. The framework comprises of a $3 \mathrm{hp} / 1440 \mathrm{rpm}$, T.E.F.C. type three-stage acceptance engine, and two voltage transformers with change proportion of $230 / 3 \mathrm{~V}$, two current.

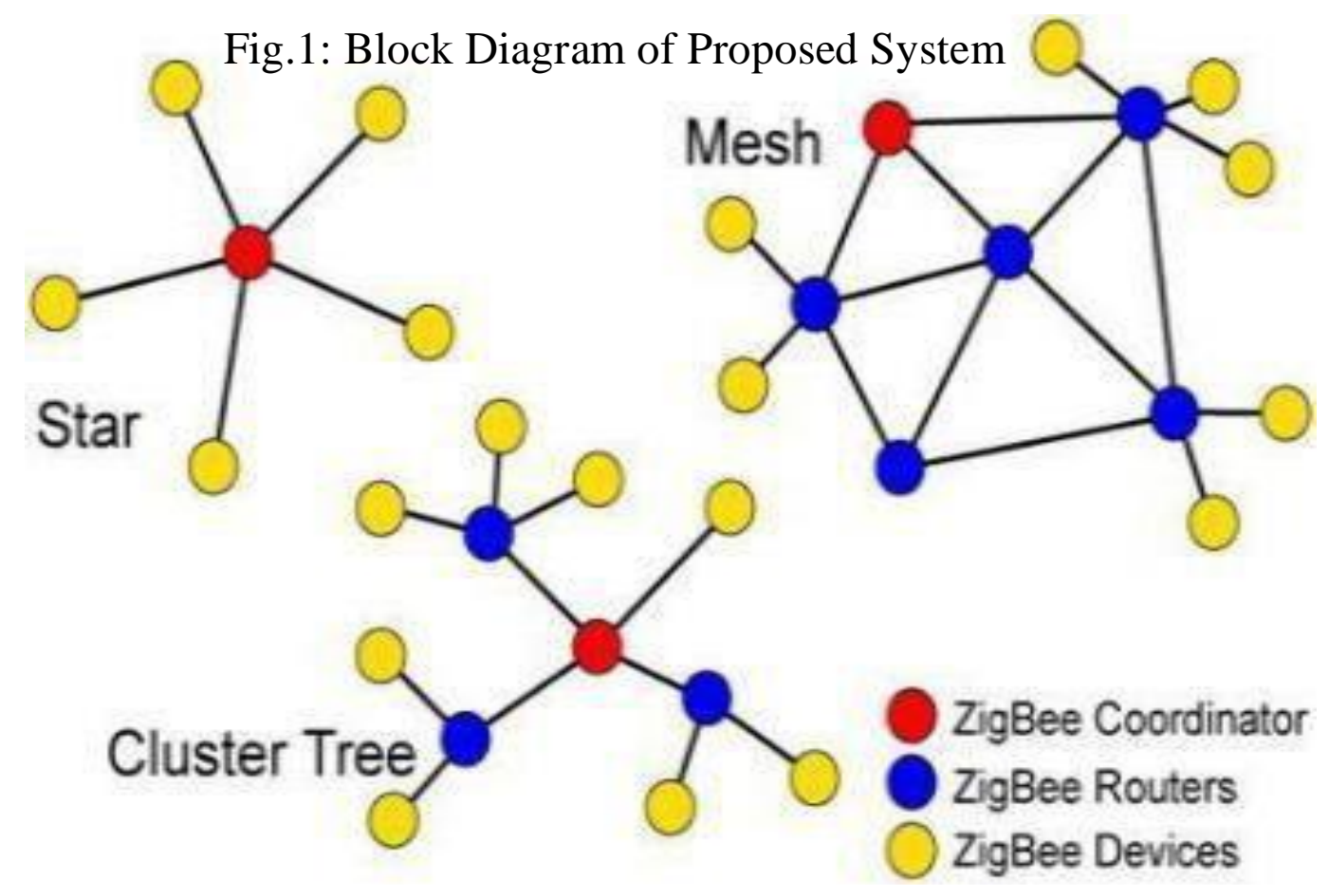


The system transferred to the Zigbee coordinator via the Zigbee router; and then, they have been transferred to the computer over the RS232 protocol. Graphical User Interface used to display received data on the screen to perform controlling commands and to storage data receive throughout the Zigbee system.

\section{IV) TEST\&RESULTS}

Five usage photos of the proposed framework are portrayed in fig. 9 to fig. 13. The fig. 9 shows the outline of the proposed framework demonstrating the force supply, starter, observing unit and acceptance engine. Fig. 10 delineates the voltage, current detecting sheets, Force supply for advanced sign regulator and Zigbee Transmitter. Fig. 11 represents the association of Zigbee Beneficiary with PC through RS232. Fig. 12 and fig. 13 are demonstrating the outcome on LCD show under burden condition. At long last fig. 13 shows the boundaries on the graphical interface demonstrating a similar outcome got as on the LCD show. All the information are put away in the neighborhood plate of PC as a dominate record as on table 1 .

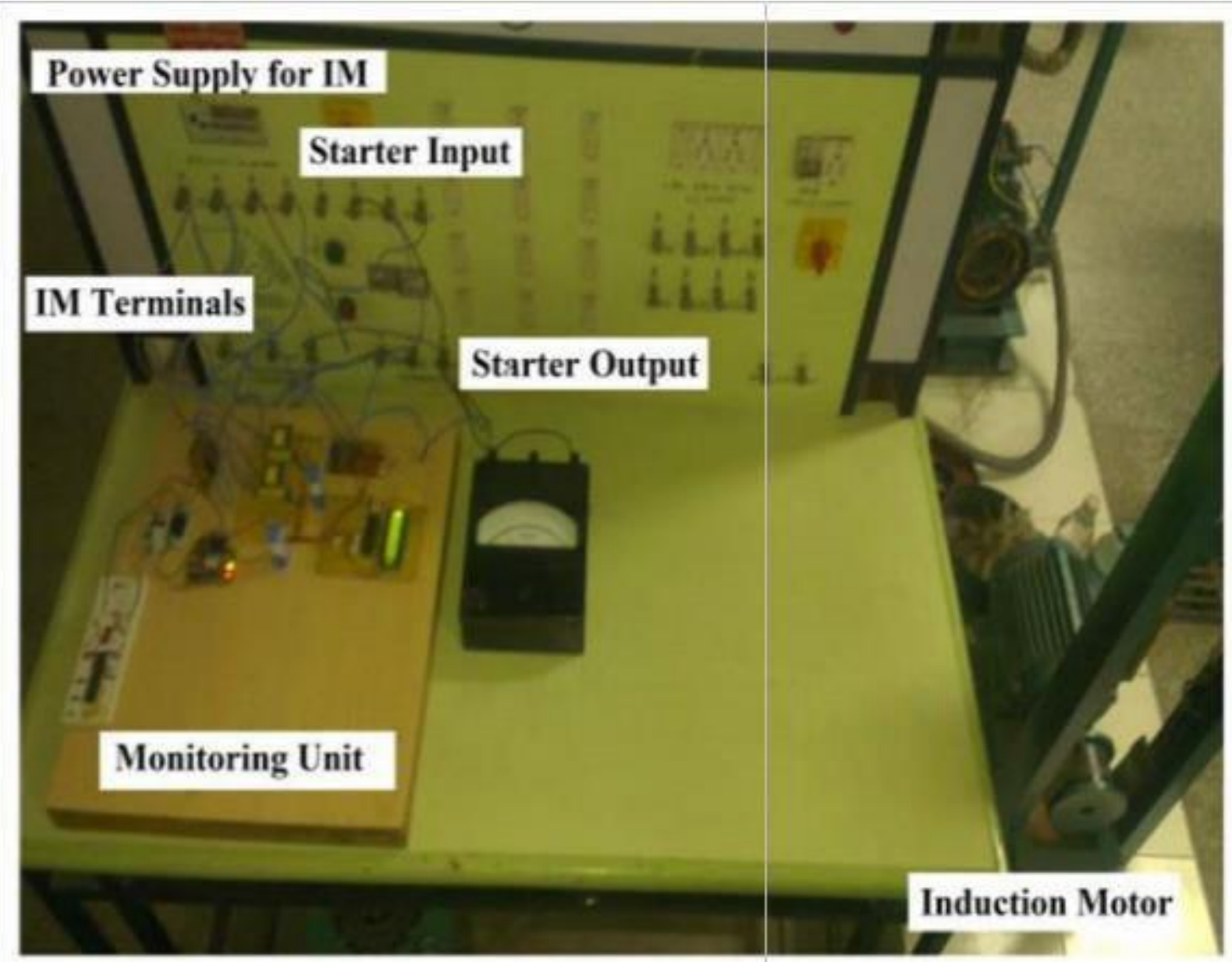

Fig. 4: overview of the proposed system 


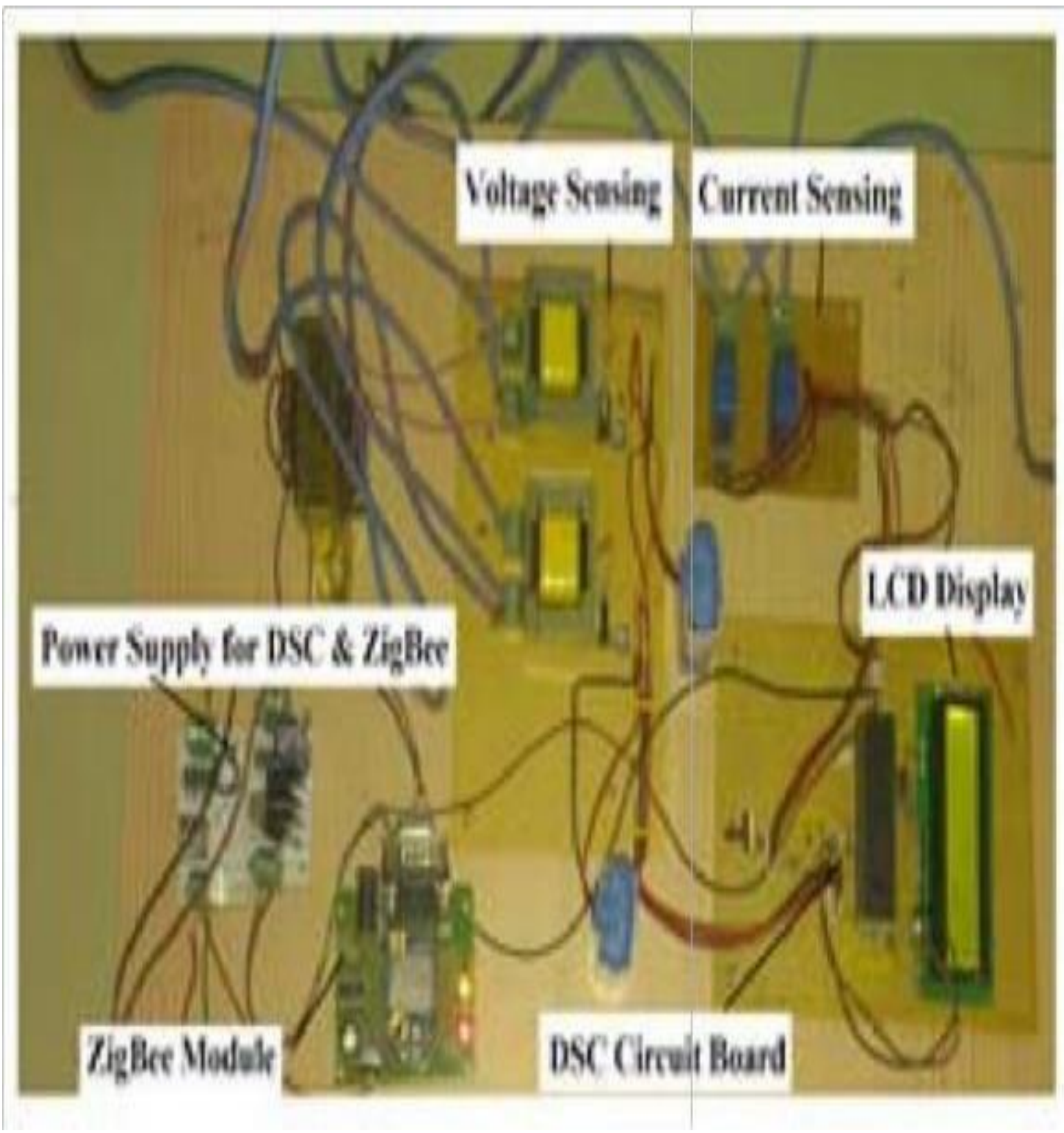

Fig. 5: Components Used 


\section{CONCLUSION}

This proposed research work is planned and actualized in Electrical Machine Lab of Electrical Designing Division at KKWIEE\&R; Nasik (India).The framework created has been tried tentatively and discovered fruitful in checking the boundaries.

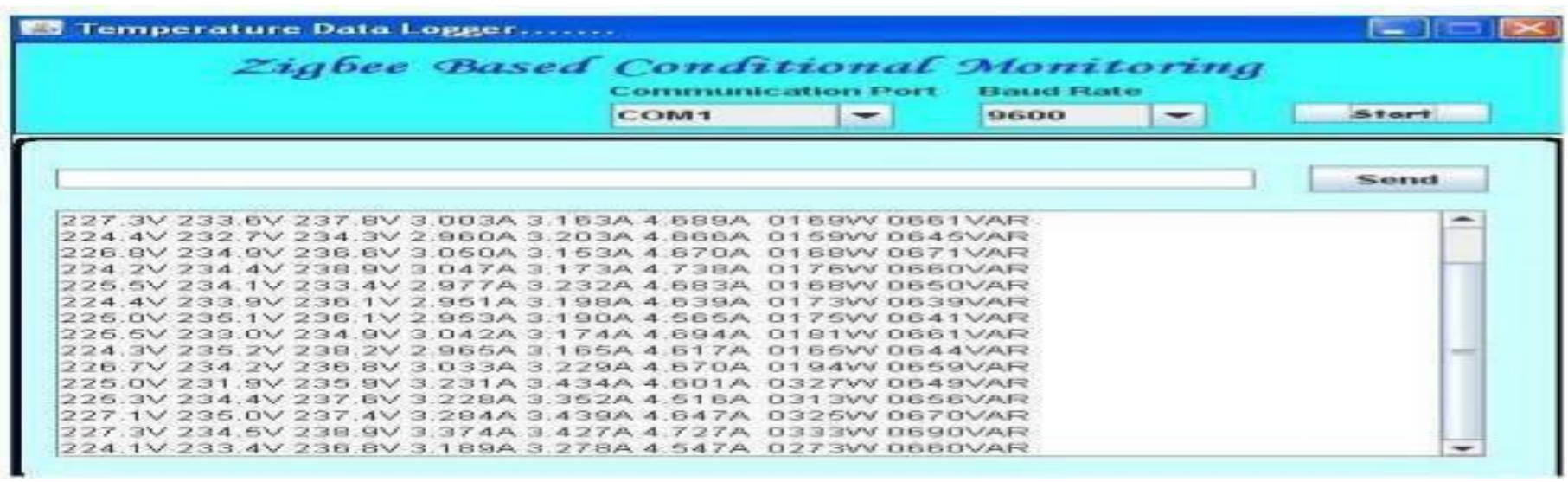

Fig.6: A view of the Results on Home Page.

\begin{tabular}{|c|c|c|c|c|c|c|c|c|}
\hline 2 & A & B & $c$ & D & E & $F$ & $G$ & $H$ \\
\hline 1 & Vrn & Vyn & Vbn & Ir & Iy & Ib & w & Var \\
\hline 2 & $227.3 \mathrm{~V}$ & $233.6 \mathrm{~V}$ & $237.8 \mathrm{~V}$ & 3.003A & $3.163 A$ & $4.689 A$ & $0169 \mathrm{~N}$ & 0661VAR \\
\hline 3 & $224.4 \mathrm{~V}$ & $232.7 v$ & $234.3 \mathrm{~V}$ & $2.960 A$ & $3.203 A$ & $4.666 A$ & $0159 \mathrm{~N}$ & O64SVAR \\
\hline 4 & $226.8 \mathrm{~V}$ & $234.9 \mathrm{~V}$ & $236.6 \mathrm{~V}$ & $3.050 A$ & 3.153A & $4.670 A$ & $0168 \mathrm{~W}$ & OGTIVAR \\
\hline 5 & $224.2 \mathrm{~V}$ & $234.4 \mathrm{~V}$ & $238.9 \mathrm{~V}$ & $3.047 A$ & $3.173 A$ & $4.738 A$ & $0176 \mathrm{~N}$ & OG6OVAR \\
\hline 6 & $225.5 \mathrm{~V}$ & $234.1 \mathrm{~V}$ & $233.4 \mathrm{~V}$ & $2.977 A$ & $3.232 A$ & $4.683 A$ & $0168 \mathrm{~W}$ & OGSOVAR \\
\hline 7 & $224.4 \mathrm{~V}$ & $233.9 \mathrm{~V}$ & $236.1 \mathrm{~V}$ & $2.951 \mathrm{~A}$ & 3.198A & $4.639 A$ & $0173 \mathrm{~W}$ & O639VAR \\
\hline 8 & $225.0 \mathrm{~V}$ & $235.1 \mathrm{~V}$ & $236.1 \mathrm{~V}$ & $2.953 A$ & $3.190 A$ & $4.565 A$ & $0175 \mathrm{~N}$ & O641VAR \\
\hline 9 & $225.5 \mathrm{~V}$ & $233.0 \mathrm{~V}$ & $234.9 \mathrm{~V}$ & $3.042 A$ & $3.174 A$ & $4.694 A$ & $0181 \mathrm{~W}$ & 0661VAR \\
\hline 10 & $224.3 \mathrm{~V}$ & $235.2 \mathrm{~V}$ & $238.2 \mathrm{~V}$ & $2.965 A$ & $3.165 A$ & $4.617 A$ & $0165 \mathrm{~W}$ & OG44VAR \\
\hline 11 & $226.7 \mathrm{~V}$ & $234.2 \mathrm{~V}$ & $236.9 \mathrm{~V}$ & $3.033 A$ & $3.229 A$ & $4.670 A$ & $0194 \mathrm{~W}$ & O659VAR \\
\hline
\end{tabular}

Table 1: Data stored in excel format.

During the observational tests, no issue has been noticed either imparting the ZigBee to the PC, or coordinating the equipment units utilized for observing the acceptance engine. The framework created can be utilized for modern applications as well as for instructive purposes; it implies, the framework might be valuable to schools that have professional, specialized, and mechanical training. Educators can utilize the framework introduced as a supporting showing instrument, and it very well may be adjusted in exploratory investigates effectively. Additionally, the exhibition of the enlistment engine can be recorded and if there should be an occurrence of terrible condition the assurance is likewise given by starter. At long last the proposed framework is savvy when contrasted with different ones.

The proposed framework is tried uniquely for voltage, current, powers, temperature with the assistance of ZigBee and work area pc/PC, however this framework can be adjusted for speed, vibration, moistness, compound boundaries checking by adding a few sensors to framework and rolling out little improvements in the program structure.

\section{VI) REFERENCES}

[1] "Intelligent Microprocessor Based Devices Provides Advanced Motor Protection.”, IEEE Conference record of annual pulp and paper industry technical Conference, Piscataway, NJ, USA, 1996, pp. 176-1 84

[2] Ramazan Bayindir, Ibrahim Sefa, 'Ilhami Colak, and Askin Bektas "Fault Detection a Protection of Induction Motors Using Sensors", IEEE transactions on energy conversion, vol.23, no. 3, September 2008.

[3] Cohen, V. and Leitert, K.h.."Motor Protection and Starting",PCIM Europe Issue 1/1997, pp.28-32

[4] FARAG, S.F. AND JHAVERI, MIKE, "A PLC based monitoring and control of power factor of a three phase induction motors", MSc Thesis, Gazi University, Institute of Science and Technology, Ankara (2009).

[5] Chengbo YU, Yanfei LIU, Cheng WANG.: Research on ZigBee Wireless Sensors Network Based on ModBus Protocol, Wireless Sensor Network, 2009, 1, 1-60.

[6] Vongsagon Boonsawat, Jurarat Ekchamanonta, Kulwadee Bumrungkhet, and Somsak Kittipiyakul: XBee Wireless Sensor Networks for Temperature Monitoring, Industrial Applications Conference, 661-667, Sept 2007.

[7] Cunkq, M., "Protection of Asynchronous Motors Aided PC by means of Microcontroller", Spring Seminar on Electronics Technology, 978- 1-4244$7850-7 / 2010 / \$ 26.00$ @ 2010

[8] Zhu XQ., Wang JM., “The research of a wireless network based on zigbee” Journal of Electronic Technology, 1:129-132 (2006). 
[9] Siddique, A., Yadava, G. S., Singh, B. A., "Review of stator fault monitoring techniques of induction motors." IEEE Transactions on Energy Conversion, 20 (1): 106-114 (2005)

[10] CUNKQ, M., "A PLC based application for induction motor protection", Journal of Polytechnic, 10 (2): 117 (2007).

[11] Siddique, A., Yadava, G. S., Singh, B. A.,"’automatic speed control of ac drive and monitoring”.piscataway, NJ, USA, 1996, pp. 176-1

[12] M. Cunkas, R. Akkaya, A. Ozturk. "Protection of AC motors by means of microcontrollers", 2000 10th Mediterranean Electrotechnical Conference. Information Technology and Electrotechnology for the Mediterranean Countries. Proceedings. MeleCon 2000 (Cat. No.00CH37099), 1998

[13] R. R. Patil, T. N. Date, B. E. Kushare. "ZigBee based parameters monitoring system for induction motor", 2014 IEEE Students' Conference on 